\title{
Cutoff Value of the Numbers of Anaplastic Cytological Features Present in Fine-Needle Aspiration Biopsy of Bone Tumors to Predict Malignancy
}

\author{
Eviana Norahmawati ${ }^{1}$, Nayla Rahmadiani ${ }^{1 *}(\mathbb{D})$, Alidha Rakhmani ${ }^{2}$ (D) Amanda Novia Regita ${ }^{3}$ (D) \\ ${ }^{1}$ Department of Anatomic Pathology, Faculty of Medicine, Universitas Brawijaya, Malang, Indonesia; ${ }^{2}$ Department of Public \\ Health, Faculty of Medicine, Universitas Brawijaya, Malang, Indonesia; ${ }^{3}$ Bachelor Program, Faculty of Medicine, Universitas \\ Brawijaya, Malang, Indonesia
}

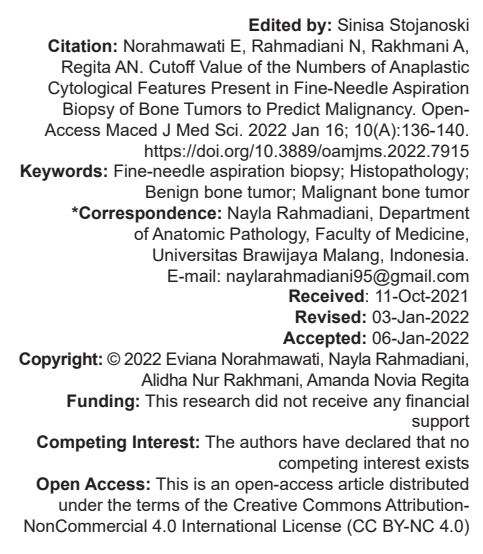

\section{Introduction}

Primary tumors of bone are relatively common; however, clinically significant bone neoplasms are infrequent. The true incidence of benign bone tumors is unknown, but bone sarcomas are rare and accounted for only $0.2 \%$ of all neoplasm [1]. The diagnosis of bone lesion can be obtained in a variety of ways, including fineneedle aspiration biopsy (FNAB), core biopsy, or open biopsy. Each of these diagnostic tools has advantages and disadvantages [2]. Initially, FNAB was used to confirm clinical suspicion of local recurrence or metastasis from neoplasm without surgical intervention. Due to its success, FNAB is developed to be the initial method to preoperative diagnosis of all tumors, both benign and malignant. FNAB has several advantages due to its simple and less invasive nature, well tolerated by patients, minimal risk of complication, ability to yield quicker results, and low cost [2], [3], [4], [5]. FNAB can also sample more easily multiple areas of a mass, potentially providing more representative material than one or two core needle biopsy specimens [3].
Although FNAB has many advantages, diagnosing and subclassifying primary bone tumors by FNAB remain one of the most challenging areas in surgical pathology and cytopathology and were limited by the high rate of specimen insufficiency, the inability to reach intramedullary lesions when the cortex is intact, and the concern for sampling and interpretation errors in the evaluation of tumors with heterogeneous or biphasic morphology [2], [3], [6]. Another limitation is due to the limited number of samples, the true condition might not be represented within the obtained samples. Furthermore, cystic lesions or heavily ossified lesions are usually suboptimal for diagnosis by FNAB [3], [7]. Considering these limitations, pathologist's experience and expertise are needed in evaluating the cytological findings in FNAB smears, because several benign and malignant bone tumors may have very similar morphology [8], [9].

The accuracy of FNAB relied heavily on the quality of the obtained samples and clinicoradiological correlation. Reported accuracy rates of FNAB for bone 
tumors range from $54 \%$ to $100 \%$ depending on the era of the study, the availability of radiologic correlation, and the diagnostic terminology used [4]. Accuracy of FNAB for the separation of benign from malignant lesions from orthopedic oncology centers utilizing FNAB was approximately 80-95\% [3].

Correlation with clinical and radiologic findings is important for the diagnosis of bone tumors. Imaging studies including plain X-ray, computerized tomography scan, and magnetic resonance imaging provide valuable information regarding the location, size, pattern of bone destruction, mineralization, periosteal reaction, and the extent of bone or soft-tissue involvement [3], [4].

Clinical practice guidelines recommend that all patients with a suspected primary malignant bone tumor be referred to a cancer center with a bone sarcoma multidisciplinary team because the approach and interpretation of biopsy demand a high level of collaboration among the members of a multidisciplinary team [4], [7].

However, in everyday practice, pathologists in Indonesia, especially in underserved area, may encounter cases with insufficient clinicoradiological data. In such cases, the diagnosis for bone tumors is determined based on the cytological findings of FNAB. Therefore, it is important to determine what cytological features in FNAB favor a benign or malignant diagnosis in bone tumors, and what is the sensitivity, specificity, and accuracy of FNAB in diagnosing bone tumor solely by evaluating the cytological features without the clinicoradiological data of the patient.

This study aims to determine the diagnostic value of cytological features of anaplasia in FNAB diagnosis of bone tumors and their ability to differentiate between benign and malignant bone tumors, and to determine the cutoff value of the numbers of anaplastic cytological features needed to be present in FNAB of bone tumors to establish a malignant diagnosis.

\section{Methods}

This is an analytical cross-sectional study conducted to 35 bone tumor cases which underwent FNAB and subsequent histopathological examination in the Department of Anatomical Pathology of Saiful Anwar General Hospital, East Java, Indonesia, within the period of January 2014-December 2017. Cases which did not have follow-up histopathological examination or yielded inconclusive result in FNAB were excluded from the study.

The complete record for the cases included in this study was obtained. All cases were previously diagnosed using histopathological examination with clinicoradiological correlation and were signed out as benign/malignant. FNAB slides of these cases were re-examined by a single investigator (EN) to evaluate the cytological findings. Cytological features observed were as follows: Characteristics of the nuclei, nucleoli, and chromatin, presence of pleomorphism, and mitosis. The investigator would note and count the presence of any of the following features of anaplasia: pleomorphism or small blue round cells, high nuclear-cytoplasmic (N/C) ratio, prominent nucleoli, hyperchromatic nuclei, clumpy and coarse chromatin, or atypical mitosis [10], [11], [12], [13]. During re-examination of FNAB slides, the investigator was blinded from patient's identity, previous histopathological diagnosis, and the respective clinicoradiological data.

Diagnostic value testing was performed using $2 \times 2$ table (using histopathological examination as the gold standard) and receiver operating characteristic (ROC) curves to obtain the sensitivity, specificity, positive predictive value (PPV), negative predictive value (NPV), and accuracy of FNAB, as well as cutoff value of the numbers of anaplastic cytological features needed to be present in FNAB of bone tumors to establish a malignant diagnosis. $p<0.05$ is considered statistically significant. All statistical analyses were performed using SPSS version 23 (IBM Corp., Armonk, NY, USA).

\section{Results}

Thirty-five cases were included in this study, which consisted of 22 malignant bone tumors and 13 benign bone tumors. Females predominated the study population. The youngest patient included in this study was 8 years old and the oldest was 63 years old. All cases were previously diagnosed using histopathological examination with clinicoradiological correlation. The characteristics of study population are described in Table 1.

Table 1: Characteristics of the study population

\begin{tabular}{lll}
\hline & Benign & Malignant \\
\hline Age (years) & $(\Sigma, \%)$ & $(\Sigma, \%)$ \\
$0-20$ & $5(38.46)$ & $10(45.45)$ \\
$21-40$ & $2(15.38)$ & $7(31.82)$ \\
$41-60$ & $4(30.77)$ & $5(22.73)$ \\
$>60$ & $2(15.39)$ & $0(0)$ \\
& Mean \pm SD (years): $35.76 \pm 19.29$ & Mean \pm SD (years): $28.09 \pm 15.96$ \\
& Oldest age (years): 63 Youngest age & Oldest age (years): 60 Youngest \\
& (years): 14 & age (years): 8 \\
Gender & $(\Sigma, \%)$ & $(\Sigma, \%)$ \\
Male & $4(30.80)$ & $11(50)$ \\
Female & $9(69.20)$ & $11(50)$ \\
Benignityl & $13(37.14)$ & $22(62.86)$ \\
malignancy & & \\
\hline
\end{tabular}

Cytological features evaluation result found that pleomorphism or small blue round cells were present in all malignant bone tumors. Other features which were also prevalent in malignant cases were hyperchromatic nuclei $(95.50 \%)$, clumpy and coarse chromatin $(86.40 \%)$, and high $\mathrm{N} / \mathrm{C}$ ratio $(72.70 \%)$. The most common cytological feature of anaplasia to be 
present in benign bone tumors was hyperchromatic nuclei $(61.50 \%)$. Meanwhile, the cytological feature of anaplasia which was absent in all benign bone tumors examined was atypical mitosis. Detailed reports of the cytological evaluation are described in Table 2.

Table 2: Cytological features evaluation result of FNAB of bone tumors

\begin{tabular}{llllll}
\hline Category & \multicolumn{2}{l}{ Benign bone tumor (\%) } & & \multicolumn{2}{c}{ Malignant bone tumor (\%) } \\
\cline { 2 - 3 } \cline { 5 - 6 } & Present & Absent & & Present & Absent \\
\hline $\begin{array}{lllll}\text { Pleomorphism or small blue } \\
\text { round cells }\end{array}$ & 7.70 & 92.30 & & 100 & 0 \\
High N/C ratio & & & & \\
Prominent nucleoli & 30.80 & 69.20 & & 72.70 & 27.30 \\
Hyperchromatic nuclei & 46.20 & 53.80 & & 40.90 & 59.10 \\
Clumpy and coarse chromatin & 61.50 & 38.50 & & 95.50 & 4.50 \\
Atypical mitosis & 15.40 & 84.60 & & 86.40 & 13.60 \\
\hline
\end{tabular}

\section{Statistical analysis}

ROC curve was generated by comparing the numbers of anaplastic cytological features present during FNAB re-examination and the previous histopathological diagnosis. ROC curve in Figure 1 showed curve far above green (50\%) and close to $100 \%$, this finding indicated that cytology scoring system in FNAB had good diagnostic value [14]. AUC value obtained from ROC curve was $96.5 \%$. Hypothesis test resulted in a $p=0.001 \quad(p<0.05)$, meaning that the AUC value of FNAB diagnosis by evaluating the numbers of anaplastic cytological features present is differ significantly with an AUC value of $50 \%$.

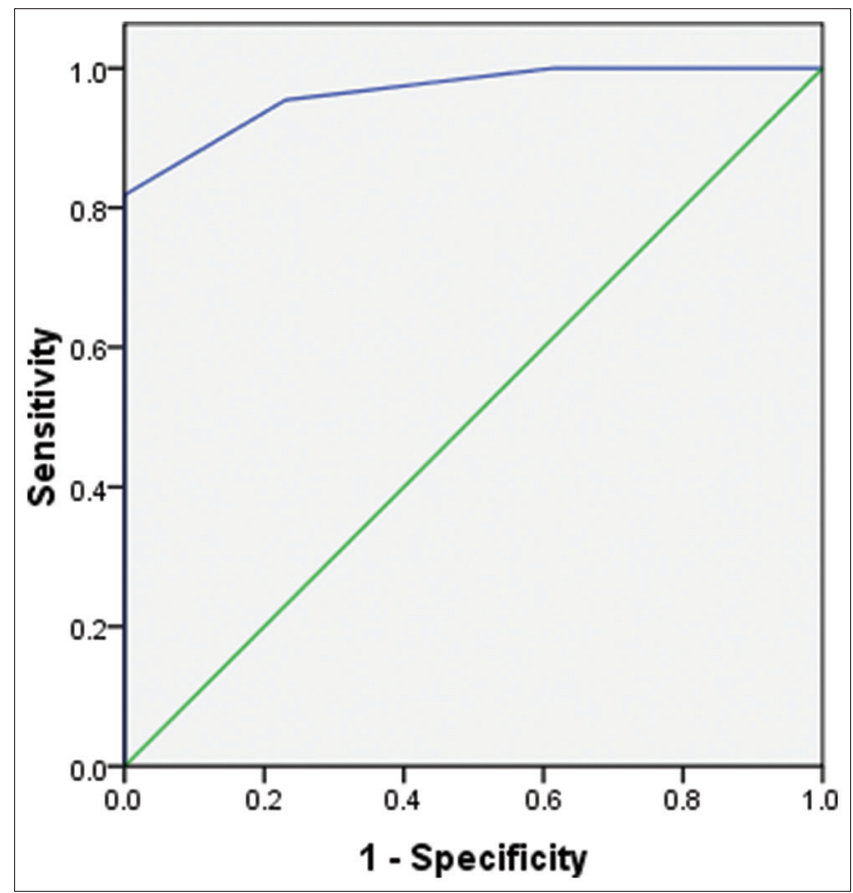

Figure 1: ROC curve

To determine the cutoff value of the numbers of anaplastic cytological features needed to be present in FNAB of bone tumors to establish a malignant diagnosis, sensitivity and specificity values from ROC curve coordinate were used [14]. Based on the $p=0.05$, a cutoff value of 2 and 4 was chosen in accordance to the sensitivity and specificity. Cutoff value of $\geq 2$ means that FNAB diagnosis of malignant bone tumor will be established if there are two or more cytological features of anaplasia; the same also apply for the cutoff value of $\geq 4$. FNAB diagnosis using 2 and 4 as the cutoff value was compared with the histopathological diagnosis using $2 \times 2$ table (Table 3 ). The results revealed that FNAB with $\geq 4$ anaplastic cytological features had $81.82 \%$ sensitivity, $100 \%$ specificity, $100 \%$ PPV, $76.47 \%$ NPV, and $88.57 \%$ accuracy. Compared with a cutoff value of $>2$, cutoff value of $>4$ showed lower sensitivity and NPV, and higher specificity, PPV, and accuracy.

Table 3: Results of the diagnostic value testing using $2 \times 2$ Table

\begin{tabular}{|c|c|c|c|c|c|}
\hline Method & $\begin{array}{l}\text { Sensitivity } \\
\text { (\%) }\end{array}$ & $\begin{array}{l}\text { Specificity } \\
\text { (\%) }\end{array}$ & PPV (\%) & $\overline{N P V ~(\%)}$ & $\begin{array}{l}\text { Accuracy } \\
(\%)\end{array}$ \\
\hline $\begin{array}{l}\text { FNAB diagnosis with the cutoff } \\
\geq 4 \text { cytological features of } \\
\text { anaplasia present }\end{array}$ & 81.82 & 100 & 100 & 76.47 & 88.57 \\
\hline $\begin{array}{l}\text { FNAB diagnosis with the cutoff } \\
\geq 2 \text { cytological features of } \\
\text { anaplasia present }\end{array}$ & 100 & 38.46 & 73.33 & 100 & 77.14 \\
\hline
\end{tabular}

\section{Discussion}

Bone tumor can occur in all ages but primary malignant bone tumor often found in younger age, especially the first two decades of life [1], [3], [13], [15]. Bone tumors tend to be location specific and most occur in a narrow age range; certain tumors are more prevalent in pediatric or adolescent patients such as osteosarcoma and Ewing sarcoma, others are more common in older adults, these include plasma cell myeloma, chondrosarcoma, chordoma, and bone metastases [1], [9]. Osteosarcoma, one of the most common malignant primary bone tumors, has a bimodal age distribution; the first peak occurs during the second decade of life and the second peak occurs in people aged $>60$ years [1], [15]. Our study population showed a wide age range, consistent with the literature.

On statistical analysis, the AUC value resulted in $96.5 \%$. Statistically, AUC value of $96.5 \%$ is considered satisfactory, which means that if the numbers of anaplastic cytological features present are used to diagnose FNAB of bone tumors, 97 out of 100 patients will get a correct conclusion. However, FNAB results in this study had lower accuracy than the previous study conducted by Akerman and Domanski [16] which showed a range of $90-97 \%$. This finding may partially be caused by the difference in sample size compared to the previous study, resulting in the discrepancy in diagnostic accuracy. Mehrota et al. [17] reported sensitivity, specificity, PPV, NPV, and accuracy of $93.3 \%, 94.5 \%, 87.5 \%, 97.2 \%$, and 
$94.2 \%$, respectively. The large variability in the reported sensitivity, specificity, and accuracy perhaps is related to the variable number of cases, the type of lesions biopsied, the presence or absence of on-site evaluation, and other factors [2]. Accuracy rates of FNAB also depend on the era of the study and the availability of clinicoradiological correlation [4].

This study also evaluated the following cytological features of anaplasia to differentiate between benign and malignant bone tumor: Pleomorphism or small round cells, high nuclear-cytoplasmic (N/C) ratio, prominent nucleoli, hyperchromatic nuclei, clumpy and coarse chromatin, or atypical mitosis. It is found that pleomorphism, high N/C ratio, prominent nucleoli, clumpy and coarse chromatin, and atypical mitosis were found in most of the malignant bone tumor group and were rarely found in benign tumor group. Atypical mitosis was not found in any of benign bone tumor cases; thus, it can be used as a reliable clue for diagnosing malignant bone tumor. Hyperchromatic nuclei and prominent nucleoli were found in most of the malignant cases, however, more than $40 \%$ of FNAB smears of benign tumors in this study also showed hyperchromatic nuclei and prominent nucleoli appearance. This finding is inconsistent with several literature and studies which stated that hyperchromatic nuclei and prominent nucleoli are present in malignant tumors and rarely found in benign tumors [10], [11], [12]. Therefore, hyperchromatic nuclei and prominent nucleoli cannot be used as a reliable diagnostic clue for pathologist to diagnose malignant bone tumor in FNAB examination. From Table 2, we can conclude that there were four cytological features which can be used as clue to differentiate between benign and malignant bone tumors: Pleomorphism, high N/C ratio, coarse and clumpy chromatin, and the presence of atypical mitosis. However, it is important to note that during the evaluation of FNAB in bone tumors, a pathologist must be aware of pitfalls such as benign lesions which mimic malignant lesions (e.g., myositis ossificans) and vice versa (e.g., parosteal osteosarcoma) [12]. Caution must be exercised on evaluation of tumors which had a uniform cell population with low pleomorphism and inconspicuous nucleoli such Ewing sarcoma [9].

Our study showed that FNAB in bone tumors with the evaluation of anaplastic cytological features had a good diagnostic value. A cutoff value of $\geq 4$ anaplastic cytological features had lower sensitivity and NPV, and higher specificity, PPV, and accuracy compared with a cutoff value of $\geq 2$. Therefore, the presence of $\geq 4$ anaplastic cytological features may aid pathologist in establishing a diagnosis using FNAB in bone tumors, especially in condition, where clinicoradiological data were insufficient. Furthermore, our study corroborated that FNAB for bone tumors can be used as initial preoperative examination in bone tumor cases to assist in subsequent diagnostic work-up or in therapeutic decision making. There are several limitations to our study. We did not assess the correlation between each feature of anaplasia with a malignant diagnosis, therefore, we cannot determine the strength of the correlation. The small sample size in our study means that the findings of our study need to be taken in the context of our study population. Future large-scale studies are needed to confirm the results of this study.

\section{Conclusions}

FNAB diagnosis of bone tumor by evaluating the presence of anaplastic cytological features without clinicoradiological data with a cutoff value of $\geq 4$ had a good sensitivity, specificity, PPV, NPV, and accuracy. Four cytological features of anaplasia were found in most malignant tumors in the study population: Pleomorphism or small blue round cell tumor, high N/C ratio, coarse and clumpy chromatin, and the presence of atypical mitosis. Further studies are needed to confirm the results of this study.

\section{Ethical Approval}

Ethical approval was obtained from Faculty of Medicine Universitas Brawijaya Research Ethics Committee: No. 142/EC/KEPK-S1-PD/04/2017.

\section{References}

1. International Agency for Research on Cancer. WHO Classification of Tumours Editorial Board. Soft Tissue and Bone Tumours. $5^{\text {th }}$ ed. Lyon, France: International Agency for Research on Cancer; 2020. p. 340-4.

2. Khalbuss WE, Parwani AV. Cytopathology of Soft Tissue and Bone Lesions. New York: Springer Science; 2011. p. 11-222.

3. Layfield LJ, Bedrossian CW, Crim JR, Palombini L. Musculoskeletal Cytohistology. Cambridge: Cambridge University Press; 2012. p. 247-74

4. Cibas ES, Ducatman BS. Cytology Diagnostic Principles and Clinical Correlates. Philadelphia, PA: Elsevier; 2019. p. 601-18.

5. Chambers M, O'Hern K, Kerr D. Fine-needle aspiration biopsy for the diagnosis of bone and soft tissue lesions: A systematic review and meta-analysis. J Am Soc Cytopathol. 2020;9(5):429-41. https://doi.org/10.1016/j.jasc.2020.05.012 PMid:32622858

6. Domanski HA, Qian X, Stanley DE. Bone. In: Domanski HA, editor. Atlas of Fine Needle Aspiration Cytology. $2^{\text {nd }}$ ed. London: Springer; 2019. p. 479-525.

7. Orell SR, Sterret G. Fine Needle Aspiration Cytology. $5^{\text {th }}$ ed. Edinburg: Churchill Livingstone Elsevier; 2012. p. 1-7.

8. Magetsari R, Marseno $\mathrm{H}$, Lanodiyu Z, Dewo P. Accuration of fine needle aspiration biopsy in musculoskeletal tumour. Int $\mathrm{J}$ 
Public Health Sci. 2016;5(2):134-6. http://doi.org/10.11591/ ijphs.v5i2.4776

9. Mody DR, Thrall MH, Krishnamurthy S. Diagnostic Pathology Cytopathology. $2^{\text {nd }}$ ed. Philadelphia, PA: Saunders Elsevier; 2018. p. 602-33.

10. Kilpatrick SE, Renner JB. Diagnostic Musculoskeletal Surgical Pathology Clinicoradiologic and Cytological Correlations. Philadelphia, PA: Saunders Elsevier; 2004.

11. Kumar V, Abbas AK, Aster JC. Robbins Pathologic Basis of Disease. $10^{\text {th }}$ ed. Philadelphia, PA: Saunders Elsevier; 2020. p. 270-4, 1171-214.

12. Bocklage TJ, Quinn RH, Schmit BP, Verschraegen CF. Bone and Soft Tissue Tumors: A Multidisciplinary Review With Case Presentations. London: JP Medical Ltd.; 2014. p. 1-21.

13. Deyrup AT, Siegal GP. Practical Orthopedic Pathology: A Diagnostic Approach. Philadelphia, PA: Elsevier; 2016.
14. Tilaki KH. Receiver operating characteristic (ROC) curve analysis for medical diagnostic test evaluation. Caspian J Intern Med. 2013;4(2):627-35.

PMid:24009950

15. Nielsen GP, Rosenberg AE, Deshpande V, Hornicek FJ, Kattapuram SV, Rosenthal DI. Diagnostic Pathology of Bone. $2^{\text {nd }}$. Philadelphia, PA: Elsevier; 2017. p. 32-3.

16. Akerman M, Domanski HA. Fine Needle Aspiration Cytology: Bone. $5^{\text {th }}$ ed. Edinburg: Churchill Livingstone Elsevier; 2012. p. 412-27.

17. Mehrotra R, Singh $M$, Singh $P$, Mannan R, Ojha V, Singh $P$. Should fine needle aspiration biopsy be the first pathological investigation in the diagnosis of a bone lesion? An algorithmic approach with review of literature. Cytojournal. 2007;4(1):9. http://doi.org/10.1186/1742-6413-4-9

PMid:17439659 